\title{
Identification of Pore Structure of Waterproof Sheet in Underground Engineering Based on Micro-CT Technology
}

\author{
Qingguo Wang, a, Qixin Yang, b \\ ${ }^{1}$ Key Laboratory of Transportation Tunnel Engineering, Ministry of Education, \\ School of Civil Engineering, Southwest Jiaotong University, Chengdu 610031, China \\ aquingguow@126.com, byangqixin113@163.com
}

\begin{abstract}
Keywords: Underground engineering; Waterproof sheet; Micro-CT technology; Porosity; Connectivity; Equivalent diameter

Abstract. The microstructure of waterproof sheet in corrosive water environment of underground engineering was studied by using X-ray CT technology, and the indexes characterizing the failure of material impermeability were explored. Distilled water and $\mathrm{NaOH}$ solutions were prepared to simulate corrosive groundwater environment, Ethylene-Propylene-Diene Monomer (EPDM) waterproof sheet in groundwater environment was scanned with micro-CT technology, 3D reconstruction images were acquired, porosity and pore connectivity were calculated, the number and volume of pores in each equivalent diameter interval were counted, and the effect on the impermeability of waterproof sheet that the changes of pore structure had was analyzed. The results show that when the difference of pore sizes is great, the largest pore will control the material impermeability. $60 \mu \mathrm{m}$ in diameter is the inflection point of the relationship between the diameter and the number of the pores. When the pore diameter is in the range between $20 \mu \mathrm{m}$ and $80 \mu \mathrm{m}$, an approximately log linear decline trend between the diameter and the number of the pores will appear. Middle and large pores have much effect on the porosity of the material. Porosity and pore connectivity can be used as the key indexes to the impermeability evaluation of waterproof sheet.
\end{abstract}

\section{Introduction}

With the largest development and utilization of underground space, it can be predictable that the study on the durability assessment and service life prediction of the structures will be on the agenda in the near future [1]. As the main materials, concrete, steel and waterproof layer compose underground structures. Waterproof layer plays an important role in preventing external groundwater percolating through concrete and corroding steel and seeping into inner space [2]. As time goes on, waterproof layer will age gradually, and ultimately lose its effectiveness, which affects the service life of the overall structure [3-6]. Therefore, it is necessary to study the damage of waterproof material in groundwater environment.

Computer tomography (CT) technology is the combination of physics and computer science. It has become an important means in micro and meso study of science due to its many advantages such as high detection efficiency, simple method, intuitive results and few empirical components. In recent years its application develops very quickly and is introduced to the field of civil engineering [7, 8].

In order to explore the indexes characterizing material impermeability, taking Ethylene-Propylene-Diene Monomer (EPDM) waterproof sheet as the subject in the study, the microstructure changes of waterproof sheet in the groundwater environment were analyzed with the help of micro-CT.

\section{Tests and methods}

Sample preparation. Referring to mass concentration or $\mathrm{pH}$ value of $\mathrm{OH}^{-}$in groundwater environment in other waterproof material tests [9], Distilled water and $\mathrm{NaOH}$ solutions were used to simulate the corrosion media in groundwater environment in the tests respectively. Mass concentration or $\mathrm{pH}$ value are shown in table 1 . 
Table 1 Mass concentration or $\mathrm{pH}$ value in the groundwater environment

\begin{tabular}{ccc}
\hline No. & Corrosive medium & Mass concentration or $\mathrm{pH}$ value \\
\hline 1 & Distilled water & - \\
2 & $\mathrm{NaOH}$ & 14 \\
\hline
\end{tabular}

EPDM was selected as test material, which was a terpolymer made from ethylene, propylene and unconjugated alkadiene. The material samples were immersed in distilled water and $\mathrm{NaOH}$ solutions for some time respectively, as shown in table 1. The samples were placed on test platform and scanned by micro-CT after being removed from corrosive solutions. The image files could be gained and the data could be analyzed.

Test equipment. MicroXCT-400 produced by Carl Zeiss X-ray Microscopy Inc. was used in micro-CT tests, as shown in Fig. 1.

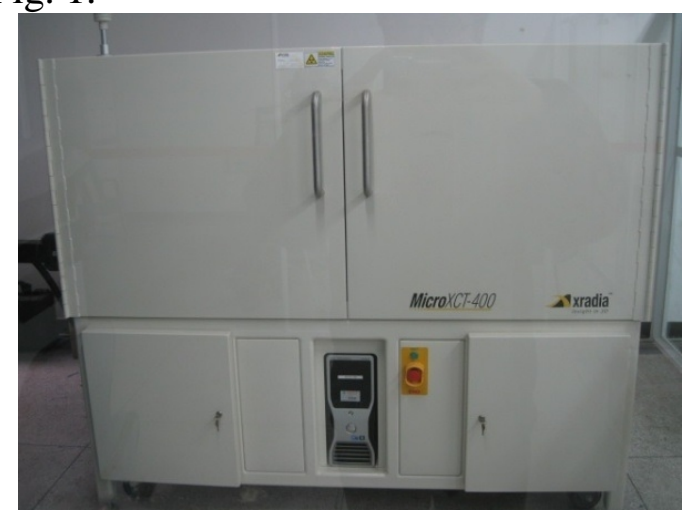

Fig. 1. MicroXCT-400

MicroXCT has best resolution and the highest contrast. It can scan the samples flexibly and provide 3D nondestructive images for quantitative analysis and a complete solution for related multi-scale research.

Different from traditional CT system whose resolution depends on small focus and high geometric magnification, MicroXCT adopts a two-stage amplification technology. First, the sample image is amplified geometrically as traditional CT system. Second, when X-rays reach the screen of the detectors, they are converted into visible lights and the image is further amplified by visible light system.

The working process of MicroXCT mainly includes search with full field and local amplification. Firstly, the whole sample is scanned with search mode, and region of interest (ROI) is distinguished and confirmed with the moderate resolution. Secondly, a virtual sample is taken locally again and the smaller region of interest is scanned with the highest resolution.

Parameters of MicroXCT used for scanning samples are shown in table 2.

Table 2 Parameters of MicroXCT used in the tests

\begin{tabular}{cccc}
\hline No. & Voltage $(\mathrm{kV})$ & Current $(\mu \mathrm{A})$ & Pixel size $(\mu \mathrm{m})$ \\
\hline 1 & 49 & 160 & 2.0721 \\
2 & 49 & 200 & 2.0485 \\
\hline
\end{tabular}

Image processing. Improving image quality is often necessary to obtain the best results with image analysis. When the samples were scanned with MicroXCT, there were CT artifacts in the images. CT artifacts referred to the difference between reconstruction data and attenuation coefficient that scanning equipment or scanned objects led to, or the case that the scanned objects did not exist but they appeared in the images [10]. CT artifacts badly affected the image quality. In order to get good analysis data on related indexes to the waterproof sheet, the artifacts had to be handled.

The main procedures for processing images were as follows [11]. First of all, the original RGB images were converted into the gray images, and the noise was weakened or eliminated by filtering and transformation. Secondly, referring to morphology characteristics in the SEM images and the equalized histograms of the gray images, segmentation thresholds of different materials and image background were acquired. Thirdly, according to the thresholds, the whole images were segmented, 
converted into the binary images and displayed in different colors respectively. Finally, the volume (area) and percentage of each material in a solid (slice) were calculated. If one pore contacted with another, separation had to be carried out prior to the statistical analysis in order to reduce the errors.

\section{Results and discussion}

In order to make a comparative study, the subsamples whose size are $400 \times 400 \times 400$ pixel $^{3}$ are captured in the center of the $3 \mathrm{D}$ reconstruction images respectively, as shown in Fig. 2. Measurement and analysis are aimed to the subsamples. If there are no special instructions, the mentioned samples all refer to the subsamples later.

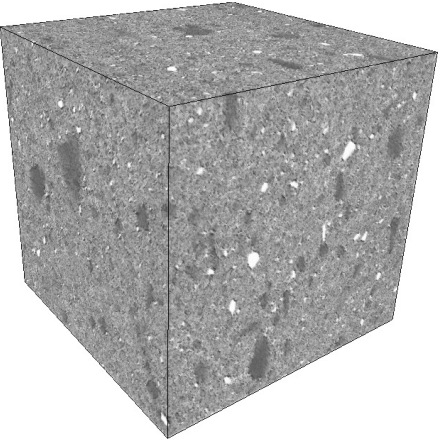

(a) Sample 1

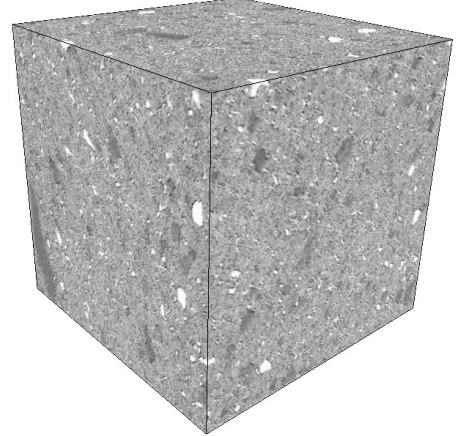

(b) Sample 2

Fig. 2. 3D reconstruction images of samples

Various material compositions in the samples can be seen from Fig. 2. The brighter the material composition is, the higher the density is, and the more the absorption of X-rays is. The bright material composition is shown in white or light grey color, and the black material composition is shown in dark grey color. The density of the bright or white spots is the highest, which are additive particles, as shown in Fig. 3. The density of the light grey material composition is the lower, which is the major constituent of the samples. The density of the dark grey material composition is the lowest, and it stands for the pores in the samples, whose distribution is shown in Fig. 4.

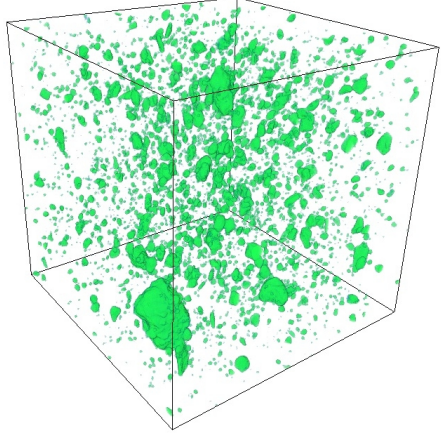

(a) Sample 1

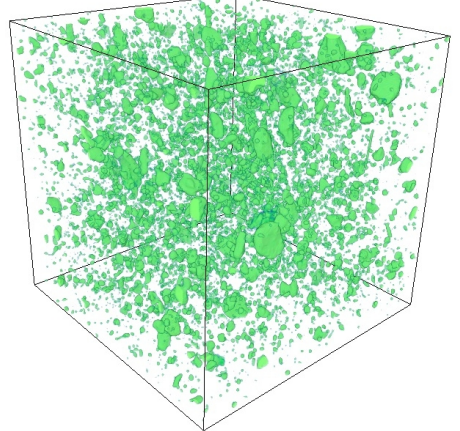

(b) Sample 2

Fig. 3. Spatial distribution of additive particles

Fig. 3 shows the spatial distribution of the additive particles in each sample. The distribution of the additive particles is disorderly and there are no obvious laws. The number and size and spatial distribution of the additive particles mainly depend on the production process and conditions of the waterproof sheet.

In order to observe the distribution of the pores in each sample intuitively, the pores are shown in different colors, as shown in Fig. 4. The number, volume, surface area, porosity and connectivity of the pores are selected as the indexes characterizing pore properties [12,13]. The indexes of the pores in each sample calculated are given in table 3. Porosity is the ratio of pore volume to total volume, and it reflects the compactness of the material. The connectivity of the large pores under certain conditions plays a dominant role on water permeation. When there is water penetration in any part, other parts are probably intact. But it means the failure of the material impermeability. In fact the 
material in other parts does not fully play its effectiveness. Considering economy, it is extremely unfavorable for the impermeability of the whole material.

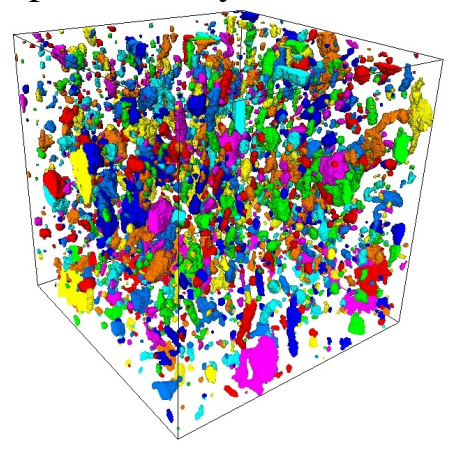

(a) Sample 1

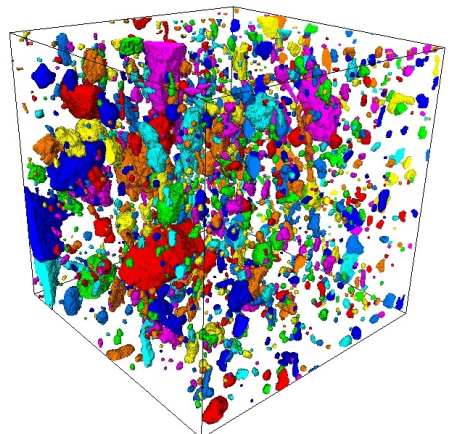

(b) Sample 2

Fig. 4. Spatial distribution of pores

Table 3 Analytical results of related pore indexes

\begin{tabular}{cccccc}
\hline No. & Number & Volume $\left(\mu \mathrm{m}^{3}\right)$ & Surface area $\left(\mu \mathrm{m}^{2}\right)$ & Porosity $(\%)$ & Connectivity $(\%)$ \\
\hline 1 & 2600 & 31145706.9 & 5688963.6 & 5.5 & 5.5 \\
2 & 1993 & 27948381.8 & 4169928.3 & 5.1 & 7.8 \\
\hline
\end{tabular}

To analyze the distributions of different size pores in the samples conveniently, the concept of equivalent diameter is introduced [14]. The formula of the equivalent diameter is extended from $2 \mathrm{D}$ to $3 \mathrm{D}$, namely that the pores are regarded as the spheres which have same volume, is defined as Eq. 1.

$$
D=\sqrt[3]{\frac{6 \times V}{\pi}}
$$

In Eq. $1 D$ and $V$ is the equivalent diameter and the volume of a single pore in the samples respectively. If there are no special instructions, all the mentioned diameters in the paper are the equivalent ones.

The optimization, mean and standard deviation of the pore diameters are given in table 4. Standard deviation is an index to the uniformity of pore sizes. The greater the degree of the uniformity is, the higher the utilization of the material is, and the stronger the material impermeability is. From table 4, the two samples have the same values basically.

\begin{tabular}{|c|c|c|c|}
\hline No. & $\operatorname{Min} / \operatorname{Max}(\mu \mathrm{m})$ & Mean $(\mu \mathrm{m})$ & StdDev $(\mu \mathrm{m})$ \\
\hline 1 & $5.9 / 148.1$ & 18.9 & 13.2 \\
\hline 2 & $5.1 / 161.0$ & 18.6 & 13.8 \\
\hline
\end{tabular}

After the pore diameter and the pore number in each sample are calculated, the pore diameter interval and the pore number are used as horizontal coordinate and vertical coordinate respectively, scatter distribution map of the pore number can be obtained, as shown in Fig. 5 and Fig. 6.

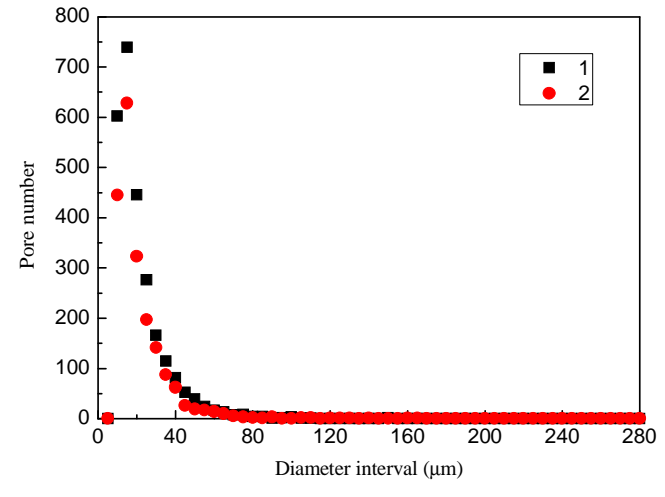

Fig. 5. Distribution of pore number

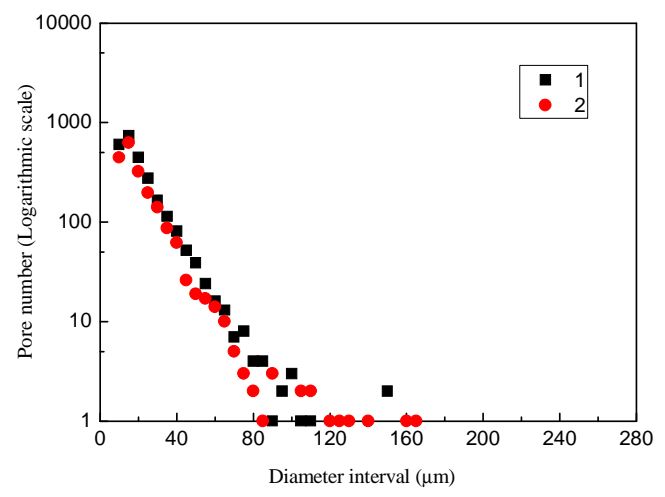

Fig. 6. Logarithmic distribution of pore number

For each sample, the number of small pores is large. The number of the pores whose diameter is less than $100 \mu \mathrm{m}$ accounts for a percentage of more than 99\%. Sample 1 and sample 2 account for a percentage of $99.8 \%$ and $99.5 \%$ respectively. 
As can be seen from Fig. 5, when the pore diameter of each sample is less than $60 \mu \mathrm{m}$, the number of the pores decreases and the decreasing rate slows as the diameter increasing. When the pore diameter is more than $60 \mu \mathrm{m}$, the number of the pores remains substantially unchanged as the diameter increasing. Fig. 6 shows logarithmic distribution map of the pore number. When the pore diameter is in the range between $20 \mu \mathrm{m}$ and $80 \mu \mathrm{m}$, an approximately log linear decline trend between the pore diameter and the pore number appears.

If the pore volume of each diameter interval in the samples is analyzed, the relationship between the pore diameter and the pore volume can be gained, as shown in Fig. 7 and Fig. 8.

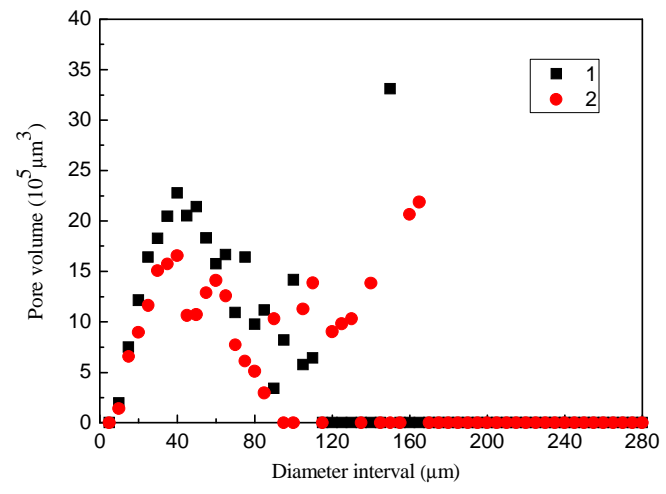

Fig. 7. Distribution of pore volume

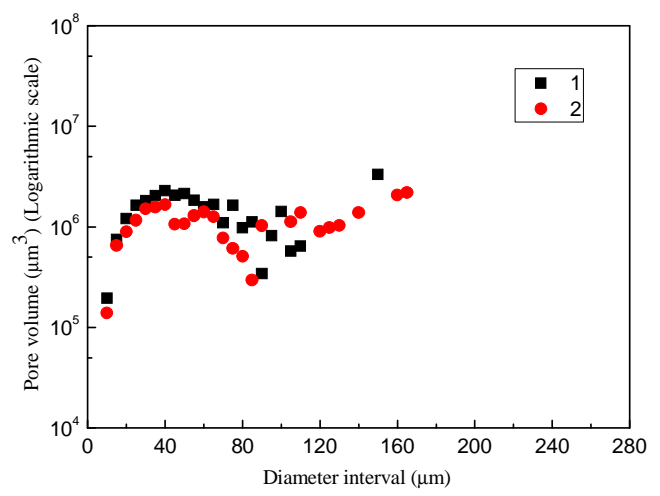

Fig. 8. Logarithmic distribution of pore volume

As can be seen from Fig. 7, the proportion of small pores in the total volume is not big, but middle and large pores play an important role on the porosity. Fig. 8 shows logarithmic distribution map of the pore volume. The logarithmic distribution map of the pore volume has the same tendency as the distribution map of that.

Pore structure includes porosity and pore connectivity. It is a key factor that has effect on mechanical, physical and chemical properties of waterproof material. When the material is immersed in corrosive solution, the total number of the pores reduces, many small pores are joined together, several large pores form, and the total volume of the pores increases. They not only reduce the material impermeability, but also weaken the mechanical property of the material. If the immersion is continued, the total volume of the pores and the pore connectivity will continuously increase. When they change to a certain extent, the pores in the material will form connected routes. It makes the material lose its effectiveness.

\section{Conclusions}

By using MicroXCT, EPDM waterproof sheet samples in different groundwater environment were scanned, 3D reconstruction images of each sample were gotten and the internal microstructures of the samples were described.

Based on the reconstruction images, the pore related indexes are calculated and analyzed. When the difference of pore sizes is great, the largest pore will control the material impermeability.

By introducing the concept of the equivalent diameter and making statistical analysis, the relationship between the pore diameter and the pore number is brought out, and the relationship between the pore diameter and the pore volume is also brought out. $60 \mu \mathrm{m}$ in diameter is the inflection point of the relationship between the diameter and the number of the pores. When the pore diameter is in the range between $20 \mu \mathrm{m}$ and $80 \mu \mathrm{m}$, an approximately log linear decline trend between the diameter and the number of pores will appear. The middle and large pores have much effect on the porosity of the material.

The method for scanning samples with micro-CT can get the internal microstructure images of the material and has the advantages of rapidity and accuracy. But the cost is high, furthermore, the method is only for the samples in groundwater environment of single corrosion factor. So the method need further development and perfection. 


\section{Acknowledgements}

The research was supported by the National Natural Science Foundation of China (Grant No. 51178401). The authors are extremely grateful to Carl Zeiss X-ray Microscopy Inc. for their help in the sample tests

\section{References}

[1] Hongke Pan, Linde Yang and Yongjing Tang: Chinese Journal of Underground Space and Engineering, 1 (2005), 804-808. (in Chinese)

[2] Changxu Ji, Xingang Wang, Kai Wang and Maotao Cai: Journal of Wuhan University of Technology, 28 (2006), 43-45. (in Chinese)

[3] Y. Yuan, X. Jiang and C. F. Lee: Tunnelling and Underground Space Technology, 15 (2000), 227-233.

[4] G. Streit and M. Achenbach: International Polymer Science and Technology, 27 (2000), 1-10.

[5] D. Raghavan and K. Egwim: Journal of Applied Polymer Science, 78 (2000), 2454-2463.

[6] Susanta Mitra, Afshin Ghanbari-Siahkali, Peter Kingshott, Kristoffer Almdal, Helle Kem Rehmeier and Anders G: Christensen. Polymer Degradation and Stability, 83 (2004), 198-206.

[7] Gengshe Yang, Dingyi Xie, Changqing Zhang and Yibin Pu: Chinese Journal of Rock Mechanics and Engineering, 15 (1996), 48-54. (in Chinese)

[8] Yingli Gao, Baixue Hu and Yangqing Luo: Highway Engineering, 35 (2010), 59-63. (in Chinese)

[9] Qixin Yang, Caoying Sheng and Xiaofeng Zhang: Modern Tunnelling Technology, 45 (2008), 39-45. (in Chinese)

[10] Shengsheng Lai and Ruimeng Yang: Journal of Mathematical Medicine, 22 (2009), 206-210. (in Chinese)

[11] Jiancheng Sun, Peifeng Zeng, Suping Yu and Xiongying Wu: Journal of Tianjin Polytechnic University, 25 (2006), 45-47. (in Chinese)

[12] S. Lu, E. N. Landis and D. T. Keane: Materials and Structures, 39 (2006), 611-620.

[13] Sheng Peng, Qinhong Hu, Stefan Dultz and Ming Zhang: Journal of Hydrology, (2012), 254-261.

[14] Guodong Shi, Deping He, Yongming Zhang and Siyuan He: Materials for Mechanical Engineering, 32 (2008), 13-15. (in Chinese) 